\title{
Simultaneous Evaluation of the Whole Aorta and Artery of Adamkiewicz by MDCT
}

\author{
Kei Takase
}

\begin{abstract}
Recent technical advancement has allowed simultaneous visualization of the artery of Adamkiewicz and whole aorta by multidetector-row-CT (MDCT). Although we could visualize the artery of Adamkiewicz in a high percentage of patients with thoracoabdominal aortic diseases, $\mathrm{CT}$ scanning with an adequate protocol and careful post-processing are necessary for accurate evaluation. Noninvasive evaluation of the artery of Adamkiewicz is useful in planning surgery. Preoperative evaluation of the intercostal arterial level from which the artery of Adamkiewicz originates is reportedly important for preventing postoperative spinal cord ischemia. Although, the usefulness of preoperative information on the artery of Adamkiewicz is still controversial, preoperative identification of the artery of Adamkiewicz by imaging has gradually spread since our first report, and has been included in preoperative evaluation items at many institutions, revealing its contribution to improvement in surgical results. (*English Translation of J Jpn Coll Angiol, 2004, 44: 693-699.)
\end{abstract}

Keywords: Adamkiewicz, aorta, MDCT, spine, aneurysm

\section{INTRODUCTION}

$\mathrm{M}$ ultidetector-row-CT (MDCT) has spread to many institutions during the 13 year period since its appearance. MDCT is particularly useful for imaging the vascular system, enabling both vascular visualization over a wide range and detailed branching diagnosis, both of which were impossible using single slice helical CT. CT was conventionally used as a method complementary to angiography, but has replaced angiography. MDCT not only replaces angiography but also provides intuitive morphological information on 3-dimensional reconstruction images. In addition, this technique has enabled the

Department of Diagnostic Radiology, Tohoku University School of Medicine, Sendai, Miyagi, Japan

Received: July 25, 2011; Accepted: September 9, 2011 Corresponding author: Kei Takase. Department of Diagnostic Radiology, Tohoku University School of Medicine, 1-1 Seiryo, Aoba-ku, Sendai, Miyagi 980-0872, Japan

Tel: +81-022-717-7312, Fax: +81-022-717-7316

E-mail:ktakase@rad.med.tohoku.ac.jp

*This article is English Translation of J Jpn Coll Angiol, 2004, 44: 693-699. visualization of blood vessels in detailed areas such as the artery of Adamkiewicz, which is often difficult to evaluate even using angiography. ${ }^{1-3)}$

Various factors are considered to be involved in the development of spinal ischemia after surgery of the thoracic or thoracoabdominal aorta. To prevent postoperative paraplegia, shortening of the spinal cord blood flow interruption time, combined use of assisted circulation (peripheral aortic perfusion, selective intercostal artery perfusion), intercostal artery reconstruction, cerebrospinal fluid drainage, and hypothermia are used. Basically, the prevention of postoperative spinal ischemia consists of the maintenance of spinal cord circulation and spinal protection. For the former, the preoperative identification of the artery of Adamkiewicz, which most markedly contributes to spinal cord blood supply in the thoracolumbar region, is useful. ${ }^{4-17)}$ Identification of the level of the intercostal or lumbar artery supplying the artery of Adamkiewicz enables the formulation of a surgical plan including the intercostal artery reconstruction level, reducing the operation time, and the preservation of the artery giving rise to the artery of Adamkiewicz or its surrounding arteries. In this study, concerning the preoperative identification of the artery of 


\section{Spinal arteries}

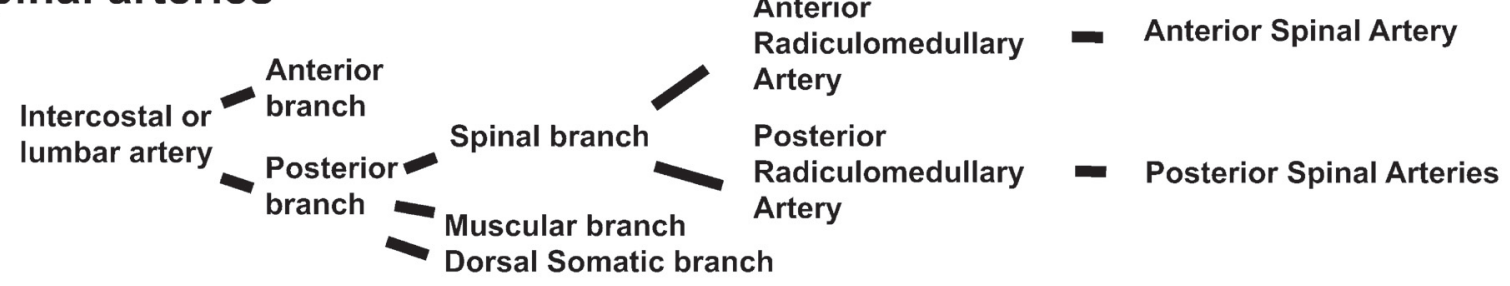

\section{Spinal veins}

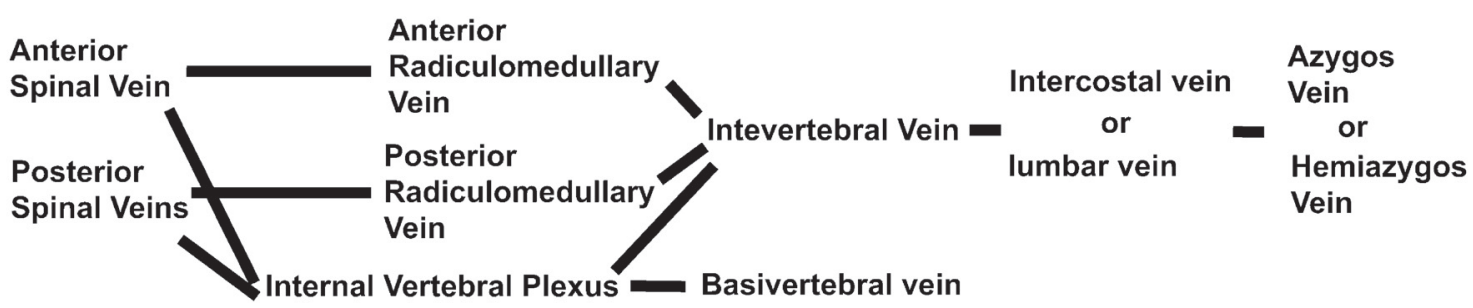

Fig. 1 Vascular anatomy of the spine.

Adamkiewicz using MDCT, the anatomy of the spinal cord arteries, and examination, image processing, and evaluation methods were described, and the clinical usefulness of visualization of the artery of Adamkiewicz was discussed.

\section{Anatomy}

The branching patterns of the spinal arteries and veins are shown in Fig. 1.

The anterior spinal artery running longitudinally along the anterior median fissure of the spinal cord supplies the anterior $2 / 3$ portion of the spinal cord, and the 2 posterior spinal arteries (one located on each side of the spinal cord posteriorly) supply the posterior funiculus. The anterior spinal artery is supplied by the anterior radiculomedullary arteries branching from the intercostal and lumbar arteries. Among the radiculomedullary arteries, the artery of Adamkiewicz is the largest. An autopsy study by Koshino et al. showed that this artery was located on the left side in $72 \%$ and between Th8 and L1 in 91\%. ${ }^{18)}$ Since the anterior spinal artery is frequently thin cranial to the entry site of the artery of Adamkiewicz and thick caudal to this site, ${ }^{19)} \mathrm{CT}$ often allows the visualization of only its caudal portion.

\section{IMAging Methods}

We devised a protocol for the simultaneous acquisition of information on the whole aorta and iliac artery and that on the artery of Adamkiewicz, and used this protocol in patients with thoracoabdominal aortic diseases for which surgery is indicated. Adequate information on major blood vessels can be obtained only by CT performed once (so-called one-stop-shop examination). Our protocol and points in the examination are introduced below. Although there are various cautionary items due to the visualization of fine structures, the procedure can be reproduced if there is a 16-(or more) detector row MDCT scanner. With an increase in the number of detector rows of MDCT, the breath-holding time decreased while scan timing became more important.

The general examination procedure is as follows:

1. Preparation before scanning

Insertion of a $20 \mathrm{G}$ indwelling needle into the right basilic vein showing a favorable condition, or into the right external jugular vein when insertion into the basilic vein is impossible

Positioning to bring the vertebral body to the isocenter

2. Non-enhanced CT scanning of major blood vessels

3. Setting of the scan initiation timing

Since most models have a function to initiate scanning after sensing contrast agent arrival at the aorta, be sure to use this function.

First, the site of the monitoring scan for contrast agent arrival at the aorta is determined.

Next, the region of interest (ROI) is established in the superior portion of the descending aorta, and the scan initiation timing is set so that scanning can be initiated 
at an intravascular CT value of about $150 \mathrm{HU}$

4. Imaging in the early phase of contrast enhancement

According to body weight, about 100-120 mL of a contrast agent at a high concentration (350-370 mgI/ $\mathrm{mL}$ ) is used. The injection rate is set at $3.5-4.5 \mathrm{~mL} / \mathrm{sec}$. A blood vessel allowing bolus injection of the contrast agent is necessary, and contrast enhancement through the right external jugular vein is often performed. For the accurate visualization of the artery of Adamkiewicz in patients with major vessel disease, a rather high dose of the contrast agent is required, but slight adjustments are made according to the body weight, renal function, and aneurysm size. It is desirable to flush with saline injection following contrast agent injection using a dual injector.

The scan range is from the lung apex to the ischial tuberosity. The scan continuation time is set at about 20 seconds. Using the finest collimation $(\leq 1 \mathrm{~mm})$ of the scanner, the electric current is set high (about $350 \mathrm{~mA}$ ).

Before the initiation of contrast enhancement, it is confirmed again using physiological saline that the contrast enhancement line allows injection without resistance or pain even at the planned injection rate, and it is explained again that patients become warm after contrast agent injection.

In some patients with aortic dissection, the artery of Adamkiewicz arises from a false lumen, or a slightly slow scan timing is appropriate. Therefore, re-scanning is performed after a breath-hold following scanning in the early phase of contrast enhancement. Although the high electric current and two scans increase the X-ray exposure dose, we permit this since the benefit of reducing the surgical risk due to the visualization of the artery of Adamkiewicz outweighs this drawback.

5. Imaging in the delayed phase

Imaging is performed to evaluate slow blood flow in the false lumen in patients with aortic dissection, the state of the walls of inflammatory aneurysms, and venous anatomy (such as the retroaortic renal vein).

6. Image reconstruction and processing

Image reconstruction using two types of field of view (FOV)

The following two types of image are produced. Shortaxis images are produced for the whole aorta without narrowing the FOV. For the straightforward identification of the artery of Adamkiewicz, the FOV is narrowed to the aorta and perispinal area, increasing spatial resolution.

\section{Image Processing on the Workstation}

We generally use the Aquarius net viewer (TeraRecon, Foster City, USA) for image interpretation, and the Ziostation (Amin, Tokyo, Japan) for detailed image processing. To readily identify the artery of Adamkiewicz on images, curved planar reformation (CPR) images from the aorta to the area along the intercostal artery, the artery of Adamkiewicz, and anterior spinal artery, is the most useful. As the previous stage of the production of this image, the artery of Adamkiewicz should be identified by detailed observation of the original animated short-axial image. ${ }^{3)}$ At the time of surgery, it is necessary to show the origin of the segmental artery (giving rise to the artery of Adamkiewicz) in the aorta using 3-dimensional images that can be straightforwardly understood by surgeons.

\section{Identification of the Artery of Adamkiewicz AND Its Visualization CAPACITY}

When reconstruction is performed at $0.5-\mathrm{mm}$ intervals, more than 1000 short-axis images are obtained. Therefore, at present, observation is performed using the paging method on the Workstation. On short-axis images, the anterior spinal artery is present on the ventral midline. The artery of Adamkiewicz enters this artery, and passes through the intervertebral foramen, running to the ventral side of the spinal cord (Fig. 2E-2H). On coronal images on cine-mode displays, since the artery of Adamkiewicz is continuous with the anterior spinal artery, forming a hair-pin curve, this structure is identified for the identification of the artery of Adamkiewicz. The level of the intervertebral foramen, through which the intercostal artery is continuous with this hair-pin curve structure, is counted from the sacral bone and regarded as the level of the artery of Adamkiewicz (Fig. 2I-2J). The most difficult problem for its identification is differentiation from the anterior radiculomedullary vein. The anterior spinal vein also shows morphology similar to that of the anterior radiculomedullary vein. Therefore, differentiation between the artery and vein is inaccurate based on morphological findings alone. The most accurate differentiation method is to visualize the continuity between the intervertebral/lumbar artery and artery of Adamkiewicz (Figs. 2I-2J). In our previous report, using 4-detector row MDCT with 2-mm collimation, we could identify the artery of Adamkiewicz in the spinal canal in $90 \%$ but could visualize the continuity between the intervertebral/lumbar artery and the artery 

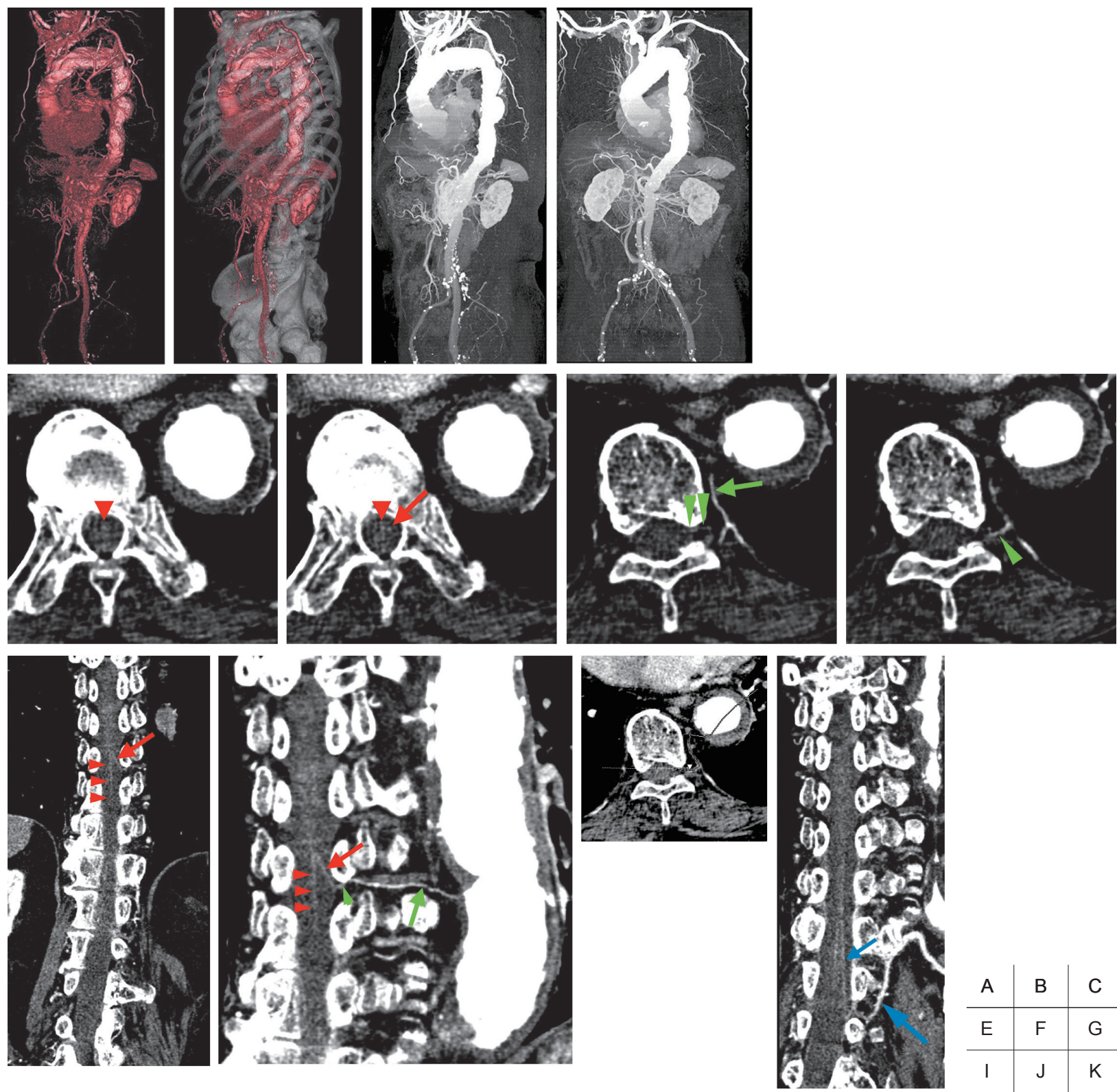

\begin{tabular}{c|c|c|c} 
A & B & $C$ & $D$ \\
\hline E & F & G & H \\
\hline I & J & K & L
\end{tabular}

Fig. 2 A male in his 70s with thoracic aortic aneurysm. 16-detector-row MDCT images with 0.5-mm collimation.

A-D: Images of the whole aorta.

A: A VR vascular image with a left anterior oblique view and (B) superimposed bone and vascular images show multiple aortic aneurysms.

C: An MIP image with a left anterior oblique view and (D) antero-posterior view shows stenosis of the right iliac artery. E-H: Transaxial images with magnified reconstruction.

The anterior spinal artery (red arrowheads) and artery of Adamkiewicz (red arrows) are clearly visualized. Continuity from the stem of the left 9th intercostal artery (green arrow), posterior branch of the intercostal artery (green arrowheads), and the artery of Adamkiewicz through the anterior spinal artery is traceable by paging method observation.

I, J: A curved planar reformation image along the aorta, left 9th intercostal artery, posterior branch of the intercostal artery, and artery of Adamkiewicz through the anterior spinal artery. Reference line is shown in $\mathbf{K}$ ).

A patent left 9th intercostal artery is clearly visualized, which supplies the artery of Adamkiewicz that continues to the anterior spinal artery, making a typical hair-pin curve.

L: A curved planar reformation image along the azygos vein, left 1st lumbar vein (large blue arrow), anterior radiculomedullary vein (small blue arrow), and anterior spinal vein.

The anterior radiculomedullary vein can be distinguished from the artery of Adamkiewicz by visualizing its continuity with venous structures, although the anterior radiculomedullary and anterior spinal veins show a similar hair-pin curve. 
Adamkiewicz in only $32 \%$ of them. In the other $68 \%$, the vessel visualized with timing not allowing venous system visualization was regarded as the artery of Adamkiewicz. ${ }^{1)}$ From our experience using 16- or 64-detector row CT with 1.0 or $0.5-\mathrm{mm}$ collimation, the artery of Adamkiewicz was visualized in about $90 \%$, which was similar to that using 4-detector row $\mathrm{CT}$, but the continuity between the intervertebral/lumbar artery and artery of Adamkiewicz could be visualized in $90 \%$ of them, showing marked improvement. Thus, differentiation between the vein and artery became possible in many cases, but we add contrast-enhanced MRA for patients in whom the visualization of the above continuity is inadequate due to adjacent bone structures. For the clear identification of the artery of Adamkiewicz, it is desirable to use a collimation $\leq 1 \mathrm{~mm}$ and a 16- or more detector row scanner. Recent studies have shown an Adamkiewicz artery visualization rate of $83-94 \%$ and its increase by improving the contrast enhancement method, reconstruction protocol function, or the intra-arterial contrast agent injection method. ${ }^{20-24)}$

\section{Case Presentation}

Patient with with the aortic arch-descending aortic aneurysm, after Y-graft replacement (Fig. 2).

In a male in his $70 \mathrm{~s}$, multiple aneurysms were present from the aortic arch to descending aorta on VR images. When original images were observed using the paging method, the anterior spinal artery and artery of Adamkiewicz were identified. A CPR image on the route from the left $9^{\text {th }}$ intervertebral artery to the anterior spinal artery was produced, and the continuity of the aorta, intercostal artery, posterior branch of the intercostal artery, the artery of Adamkiewicz, and anterior spinal artery could be observed on one image. The continuity could be traced even in the area showing passage through the intervertebral foramen. Due to improvement in resolution in the Z-axis direction, the visualization of vascular continuity improved, and the anterior radiculomedullary vein entering the left L1 intercostal vein could be identified in this patient. Entry of this vessel into the azygos venous system confirmed on the CPR image allowed differentiation from arteries.

\section{Impact of the Preoperative Identification of the Artery of Adamkiewicz on Surgery}

The successful identification of the intercostal artery that should be reconstructed is beneficial to surgical planning and the operation itself. The usefulness of selective angiography for preoperatively identifying the artery of Adamkiewicz has also been reported, ${ }^{6-9)}$ but complications often develop. In patients with thoracoabdominal aortic aneurysms or dissection, angiography of the intercostal or lumbar artery itself is almost impossible. There are only a few institutions where the artery of Adamkiewicz is preoperatively identified using angiography. Recent studies have shown noninvasive visualization of the artery of Adamkiewicz using MRI or MDCT, 1, 2, 25) and its usefulness for surgery. ${ }^{13-17)}$ The usefulness of the preoperative evaluation of the artery of Adamkiewicz has been re-evaluated.

In the development of postoperative paraplegia, not only the presence or absence of the reconstruction of the artery of Adamkiewicz but also various factors such as the intraoperative arterial cross-clamping time, intraoperative perfusion method, and individual differences in spinal cord hemodynamics may be involved. However, the preoperative clarification of the origin of the artery of Adamkiewicz and reconstruction of only the intercostal/ lumbar artery as its supplying blood vessel shorten the intraoperative arterial cross-clamping time, ${ }^{13,15)}$ and may contribute to the accurate preservation of postoperative blood flow in the artery of Adamkiewicz. If the artery of Adamkiewicz can be confirmed to be located outside the range allowing artery replacement, intercostal artery reconstruction can be omitted, which shortens the operation time. ${ }^{14)}$ In addition, intraoperative selective perfusion at the level of this artery to minimize intraoperative spinal cord ischemia has been evaluated. ${ }^{16)}$ When the artery of Adamkiewicz arises from the aortic replacement range, the intercostal artery for reconstruction can be identified before surgery, and the operation time can be shortened compared with the surgical method achieving reconstruction of as many intercostal arteries as possible. ${ }^{15,17)}$ Since the intraoperative arterial cross-clamping time is associated with postoperative complications, its shortening is a major benefit.

The identification of the artery of Adamkiewicz has the above benefits. A previous study showed a significantly lower complication rate in patients in whom the artery of Adamkiewicz could be preoperatively identified using MRA, and only the intercostal artery at this level was reconstructed in those in whom many intercostal arteries were reconstructed without preoperative identification of this artery in the same institution. ${ }^{13)}$

In contrast, Griepp et al. ligated intercostal arteries showing no problems under intraoperative monitoring of somatosensory-evoked potentials, and reconstructed 
others. As a result, intercostal artery reconstruction was not necessary in any patient. They also reported no spinal cord complications after replacement of less than 10 segments but development of paraplegia in 2 of 21 patients after replacement of 10 segments or more, and an overall incidence of paraplegia of $2 \%$. Based on these results, they speculated that the anterior spinal artery does not depend on the artery of Adamkiewicz alone in terms of hemodynamics. ${ }^{26}$ )

The association between the preoperative identification of the artery of Adamkiewicz and surgical results is still controversial. This is partly because only a few years have passed since the establishment of the Adamkiewicz artery evaluation method. Even Griepp et al. hoped for the development of a safe preoperative Adamkiewicz artery identification method in the discussion of the above study in $1996 .^{26)}$

In patients with aortic diseases requiring the visualization of the artery of Adamkiewicz, occlusion of the origin of the segmental artery and blood supply to the artery of Adamkiewicz by collateral circulation are often present. Using our simultaneous visualization protocol, collateral circulation between segmental arteries or from the internal thoracic artery can be sometimes visualized using CT, and the blood vessels for reconstruction or preservation can be more accurately determined, which may further improve the surgical results. Recently, in cooperation with the department of cardiovascular surgery, we initiated a method in which vascular reconstruction is performed after functional identification of the vessel most markedly contributing to the blood supply to the spinal cord using intraoperative perfusion cooling of the artery of Adamkiewicz using the supplying vessel suggested by MDCT. No spinal cord complications associated with this method have been observed to the present. Postoperative spinal cord complications cannot be avoided solely by preserving or reconstructing the artery of Adamkiewicz visualized using $\mathrm{CT}$, but may be associated with multiple factors. However, the complication rate can be reduced by identifying the supplying vessel based on morphological information on the artery of Adamkiewicz obtained using MDCT and intraoperative functional examination, and the combined use of intraoperative spinal cord protection methods such as spinal cord drainage and cooling.

Preoperative identification of the artery of Adamkiewicz by imaging has gradually spread since our first report using CT in 2002, ${ }^{1)}$ and has been included in preoperative evaluation items at many institutions. We expect that its contribution to improvement in surgical results and methods to avoid postoperative spinal cord complications will be shown by studies in many cases.

\section{REFERENCES}

1) Takase K, Sawamura Y, Igarashi K, et al. Demonstration of the artery of Adamkiewicz at multi- detector row helical CT. Radiology 2002; 223: 39-45. [Medline] [CrossRef]

2) Yoshioka K, Niinuma H, Ohira A, et al. MR angiography and $\mathrm{CT}$ angiography of the artery of Adamkiewicz: noninvasive preoperative assessment of thoracoabdominal aortic aneurysm. Radiographics 2003; 23: 1215-25. [Medline] [CrossRef]

3) Edited by Takase K, written by Chiba $Y$ and Takase $K$ "Manual for 3-dimensional medical image production - from the basics of Zio M900 to its clinical utilization method -"Medical View -2004.

4) Svenson LG, Crawford ES. Cardiovascular and vascular disease of the aorta. Philadelphia: WB Saunders: 1997.

5) Svensson LG, Crawford ES, Hess KR, et al. Experience with 1509 patients undergoing thoracoabdominal aortic operations. J Vasc Surg 1993; 17: 357-68, discussion 368-70. [Medline] [CrossRef]

6) Koroshetz AM, Taveras JM. Anatomy of the vertebrae and spinal cord. In: Taveras JM, ed. Radiology: Diagnosis-Imaging-Intervention. Vol 10, 101 Philadelphia, J. B. Lippincott Company. 1989; 1-14.

7) Benson JE, Han JS. Examination of the spine. In: Taveras JM, ed. Radiology: Diagnosis-ImagingIntervention. Vol 10, 102 Philadelphia, J. B. Lippincott Company. 1989; 1-18.

8) Wan IY, Angelini GD, Bryan AJ, et al. Prevention of spinal cord ischaemia during descending thoracic and thoracoabdominal aortic surgery. Eur J Cardiothorac Surg 2001; 19: 203-13. [Medline] [CrossRef]

9) Williams GM, Perler BA, Burdick JF, et al. Angiographic localization of spinal cord blood supply and its relationship to postoperative paraplegia. J Vasc Surg 1991; 13: 23-33, discussion 33-5. [Medline] [CrossRef]

10) Fereshetian A, Kadir S, Kaufman SL, et al. Digital subtraction spinal cord angiography in patients undergoing thoracic aneurysm surgery. Cardiovasc Intervent Radiol 1989; 12: 7-9. [Medline] [CrossRef]

11) Savader SJ, Williams GM, Trerotola SO, et al. Preoperative spinal artery localization and its relationship to postoperative neurologic complications. Radiology 1993; 189: 165-71. [Medline]

12) Heinemann MK, Brassel F, Herzog $T$, et al. The role of spinal angiography in operations on the thoracic aorta: myth or reality? Ann Thorac Surg 1998; 65: 346-51. [Medline] [CrossRef]

13) Kawaharada N, Morishita K, Fukada J, et al. Thoracoabdominal or descending aortic aneurysm repair after preoperative demonstration of the Adamkiewicz artery by magnetic resonance angiography. Eur J Car- 
diothorac Surg 2002; 21: 970-4. [Medline] [CrossRef]

14) Fukada J, Morishita K, Hyodoh H, et al. Descending or thoracoabdominal aortic aneurysm repair without intercostal vessel reconstruction using contrast magnetic resonance angiography: report of two cases. Surg Today 2002; 32: 163-6. [Medline] [CrossRef]

15) Fukada J, Morishita K, Kawaharada N, et al. Lessinvasive thoracic aortic aneurysm repair. Ann Thorac Surg 2002; 74: 1244-6. [Medline] [CrossRef]

16) Ohtsubo $S$, Itoh $T$, Okazaki $Y$, et al. Selective perfusion of preoperatively identified artery of Adamkiewicz during repair of thoracoabdominal aortic aneurysm. J Thorac Cardiovasc Surg 2004; 127: 272-4. [Medline] [CrossRef]

17) Hachiro Y, Kawaharada N, Morishita K, et al. Thoracoabdominal aortic aneurysm repair after detection of the Adamkiewicz artery by magnetic resonance angiography; a way to shorten operating time and improve outcome. Kyobu Geka 2004; 57: 280-3 (in Japanese). [Medline]

18) Koshino T, Murakami G, Morishita K, et al. Does the Adamkiewicz artery originate from the larger segmental arteries? J Thorac Cardiovasc Surg 1999; 117: 898-905. [Medline] [CrossRef]

19) Morishita K, Murakami G, Fujisawa Y, et al. Anatomical study of blood supply to the spinal cord. Ann Thorac Surg 2003; 76: 1967-71. [Medline] [CrossRef]

20) Backes WH, Nijenhuis RJ, Mess WH, et al. Magnetic resonance angiography of collateral blood supply to spinal cord in thoracic and thoracoabdominal aortic aneurysm patients. J Vasc Surg 2008; 48: 261-71 Epub
2008 Jun 20. [Medline] [CrossRef]

21) Nakayama $Y$, Awai K, Yanaga $Y$, et al. Optimal contrast medium injection protocols for the depiction of the Adamkiewicz artery using 64-detector CT angiography. Clin Radiol 2008; 63: 880-7 Epub 2008 Apr 14. [Medline] [CrossRef]

22) Nojiri J, Matsumoto K, Kato A, et al. The Adamkiewicz artery: demonstration by intra-arterial computed tomographic angiography. Eur J Cardiothorac Surg 2007; 31: 249-55 Epub 2006 Dec 19. [Medline] [CrossRef]

23) Takase K, Akasaka J, Sawamura Y, et al. Preoperative MDCT evaluation of the artery of Adamkiewicz and its origin. J Comput Assist Tomogr 2006; 30: 716-22. [Medline] [CrossRef]

24) Utsunomiya D, Yamashita Y, Okumura S, et al. 1. Demonstration of the Adamkiewicz artery in patients with descending or thoracoabdominal aortic aneurysm: optimization of contrast-medium application for 64-detector-row CT angiography. Eur Radiol 2008; 18: 2684-90 Epub 2008 May 29. [Medline] [CrossRef]

25) Yamada N, Takamiya M, Kuribayashi S, et al. MRA of the Adamkiewicz artery: a preoperative study for thoracic aortic aneurysm. J Comput Assist Tomogr 2000; 24: 362-8. [Medline] [CrossRef]

26) Griepp RB, Ergin MA, Galla JD, et al. Looking for the artery of Adamkiewicz: a quest to minimize paraplegia after operations for aneurysms of the descending thoracic and thoracoabdominal aorta. J Thorac Cardiovasc Surg 1996; 112: 1202-13, discussion 1213-5. [Medline] [CrossRef] 